\title{
Numerical Simulation of Explosive Combustion Following Ignition of a Fuel Vapor Cloud
}

\author{
ZHIXIN HU and ARNAUD TROUVE \\ Department of Fire Protection Engineering \\ University of Maryland \\ College Park, Maryland 20742, USA
}

\begin{abstract}
The objective of the present study is to examine the feasibility of a Large Eddy Simulation (LES) approach combined with a partially-premixed combustion (PPC) model for simulations of transient combustion events occurring in fuel vapor clouds. The PPC formulation uses: a premixed combustion sub-model based on the filtered reaction progress variable approach; a non-premixed combustion sub-model based on the Eddy Dissipation Concept; and a premixed/non-premixed combustion coupling interface based on the concept of a flame index. The PPC model is implemented into the Fire Dynamics Simulator (FDS) developed by the National Institute of Standards and Technology, USA. Because FDS uses an incompressible flow solver, the present study is restricted to combustion scenarios featuring low Mach numbers (e.g., scenarios with no blast wave). The enhanced FDS modeling capability is evaluated by detailed comparisons with an experimental database previously developed by FM Global Research, USA. The test configuration corresponds to controlled ignition followed by explosive combustion in an enclosure filled with vertically-stratified mixtures of propane in air, both with and without venting, and with and without obstacles. All studied cases develop significant compartment over-pressures; these pressurized combustion cases present a particular challenge to the bulk pressure algorithm in FDS which has robustness and accuracy issues, in particular in vented configurations. The FDS bulk pressure algorithm is modified in the present study in order to allow detailed comparisons between measured and simulated pressure time histories. Overall, the comparison between numerical results and experimental data ranges from fair to good, and confirms the feasibility of a LES treatment of explosive combustion.
\end{abstract}

KEYWORDS: fire modeling, CFD, explosion, deflagration, partially premixed combustion.

\section{INTRODUCTION}

The present study is motivated by fire and explosion safety questions following the accidental release and possible subsequent ignition of vaporized fuel in ambient air [1-5]. Such questions are asked when examining a number of real-world fire hazards, for instance spilling/leaking fuel tank or fuel pipe scenarios, mining accidents, and backdraft scenarios. We assume in the following that the fuel release takes place in ambient air, and that there is a significant delay between the start of the fuel build-up and the ignition event, thereby allowing the formation of a sizeable fuel vapor cloud prior to combustion. Depending on the velocity of the fuel-air mixing process, the composition of the bulk of the fuel vapor cloud will be ultralean (i.e., below the lower fuel-air flammability limit) in the case of fast mixing, ultra-rich (i.e., above the upper flammability limit) in the case of slow mixing (Fig. 1), or flammable (i.e., within the flammability limits) in the intermediate case (Fig. 2). The fast mixing case corresponds to a desirable safe dispersion scenario in which there is no fire or explosion hazard; we focus in the present study on the slow mixing and intermediate cases that correspond to a hazard (Figs. 1a-2a).

We further assume that ignition takes place at some flammable location in the fuel vapor cloud (Figs. 1b$2 \mathrm{~b})$. Following ignition, the combustion will proceed initially as a thin deflagration or detonation wave that propagates across the flammable portions of the fuel vapor cloud. We focus in the following on the deflagration scenario, in which the premixed flame propagates at subsonic speeds and pressure remains quasi-uniform across the combustion zone (pressure may change with time but not with spatial location). Even with this limited scope, the combustion dynamics remain quite complex since they depend strongly on the state of the fuel-air mixing field found at ignition time. In the case of an ultra-rich fuel vapor cloud, combustion corresponds predominantly to a diffusion burning mode (Fig. 1c); in the case of a flammable fuel vapor cloud, combustion includes an intense premixed burning mode (Fig. 2c). In cases with 


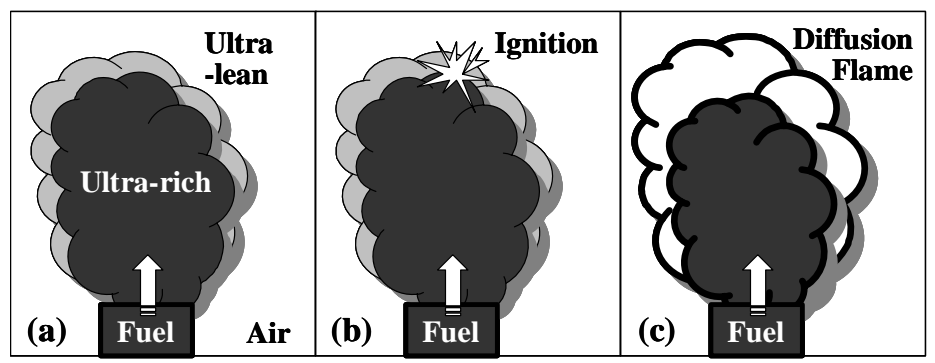

Fig. 1. Problem configuration corresponding to (a) the formation of a large ultra-rich fuel vapor cloud, followed by (b) ignition and (c) diffusion burning.

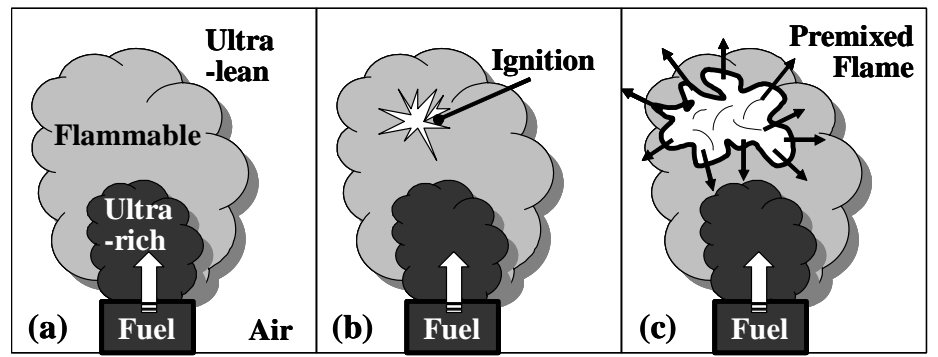

Fig. 2. Problem configuration corresponding to (a) the formation of a large flammable fuel vapor cloud, followed by (b) ignition and (c) deflagration.

significant premixed burning, one may also differentiate between pure premixed and partially-premixed burning modes. Pure premixed burning modes are observed when the bulk of the fuel vapor cloud is flammable fuel-lean, while partially-premixed burning modes are observed when some portions of the fuel vapor cloud are flammable fuel-rich (in that case, the combustion starts as a fuel-rich deflagration wave and propagates across the flammable region while leaving excess fuel in the post-deflagration gases; the residual fuel may then subsequently mix with ambient air and burn in a diffusion flame mode).

Our objective in the present study is to examine the feasibility of a Computational Fluid Dynamics (CFD) approach to simulate the range of scenarios illustrated in Figs. 1-2. The subject of accidental combustion in fuel vapor clouds has received significant interest in the scientific literature. Previous CFD modeling studies typically belong to one of the following two categories: studies in which flammable conditions are assumed across the bulk of the fuel vapor cloud, and combustion is described as premixed [6-11]; and studies in which ultra-rich conditions are assumed and combustion is described as non-premixed [12-16]. Clearly the ignition/deflagration/diffusion-flame scenarios presented in Figs. 1 and 2 require a more general formulation in which combustion can be described as both, simultaneously or sequentially, premixed and non-premixed.

The present study considers such a formulation and focuses on specific issues resulting from the coupling of premixed and non-premixed turbulent flame models. This coupling has received growing interest in recent years, primarily driven by the need to adapt combustion formulations for a CFD treatment of lifted turbulent diffusion flames [17-19]. The burning regime in the stabilization region of lifted diffusion flames is usually referred to as partially-premixed combustion (PPC). The present study may be viewed as a continuation of the PPC modeling work in Refs. [18-19].

The developments and tests presented herein are made in the context of a CFD solver called the Fire Dynamics Simulator (FDS). FDS is developed by the National Institute of Standards and Technology (NIST), USA, and is oriented towards fire applications; it uses a Large Eddy Simulation (LES) approach for turbulence (based on the classical Smagorinsky model) and a fast chemistry model for non-premixed combustion (based on the Eddy Dissipation Concept) [20-21]. A premixed combustion modeling capability has also been recently incorporated into FDS [22-25]. The present study is a continuation of the work presented in Refs. [22-25], an extension to FDS Version 5 (earlier developments had been made in the context of FDS Version 4), as well as a new validation study that uses an experimental database previously developed by FM Global Research, USA. 


\section{MODELING OF PARTIALLY-PREMIXED COMBUSTION}

\section{Deflagration Modeling}

We start from the classical description of premixed combustion based on the concept of a reaction progress variable $c$ : $c=0$ in the fresh reactants, $c=1$ in the burnt products, and the flame is the region where $c$ goes from 0 to 1 (see Refs. [22-25] for additional details and entries to the deflagration modeling literature). The $c$-framework is general and flexible, and it has been previously adapted to a LES treatment of propagating turbulent flames. The treatment is based on a transport equation for the LES-filtered reaction progress variable $\widetilde{c}$. We adopt in the following the closure models of Refs. [26-27] and write:

$$
\frac{\partial}{\partial t}(\tilde{\rho} \tilde{c})+\frac{\partial}{\partial x_{i}}\left(\bar{\rho} \tilde{u}_{i} \tilde{c}\right)=\frac{\partial}{\partial x_{i}}\left(\left(\bar{\rho} \frac{v_{t}}{S c_{t}}+\frac{\rho_{u} s_{L} \Delta_{c}}{16 \sqrt{6 / \pi}}\right) \frac{\partial \tilde{c}}{\partial x_{i}}\right)+4 \rho_{u} s_{L} \Xi \sqrt{\frac{6}{\pi}} \frac{\tilde{c}(1-\tilde{c})}{\Delta_{c}}+\overline{\dot{\omega}_{i g n}^{\prime \prime \prime}}
$$

where $\rho$ is the mass density, $u_{i}$ the $x_{i}$-component of the flow velocity vector, $v_{t}$ the turbulent eddydiffusivity, $S c_{t}$ a turbulent Schmidt number, $\rho_{u}$ the unburnt gas mass density, $s_{L}$ the laminar flame speed, $\Delta_{c}$ the LES $c$-filter size, $\Xi$ the subgrid-scale flame wrinkling factor, $\overline{\dot{\omega}_{i g n}^{\prime \prime \prime}}$ an extra source term, and where the over-bar (tilde) symbol denotes straight (Favre-weighted) LES-filtered quantities. The first term on the right-hand-side of Eq. (1) represents subgrid-scale convective transport and molecular diffusion transport of $c$; the second term represents production of $c$ due to chemical reaction; the last term represents ignition. The subgrid-scale convective transport term has been expressed assuming gradient-transport and using the classical turbulent eddy viscosity concept. The molecular diffusion term has been expressed according to the realizability requirement that under laminar flow conditions, the flame propagates at the laminar flame speed $s_{L}$ [22,26-27]. The chemical reaction term $\overline{\dot{\omega}}_{C}$ has been expressed using a classical flamelet closure expression. While elaborate closure model expressions are available in the scientific literature to describe the subgrid-scale flame wrinkling factor, we choose in the present study to treat $\Xi$ as a model coefficient (see the results Section).

The corresponding expression for the LES-filtered fuel mass reaction rate (in units of $\mathrm{kg} / \mathrm{s} / \mathrm{m}^{3}$ ) is:

$$
\overline{\dot{\omega}_{p}^{\prime \prime \prime}}=\left(4 \rho_{u} s_{L} \Xi \sqrt{\frac{6}{\pi}} \frac{\widetilde{c}(1-\tilde{c})}{\Delta_{c}}+\overline{\dot{\omega}_{i g n}^{\prime \prime \prime}}\right) \times\left(Y_{F}^{m}-Y_{F}^{e q}\right)
$$

where $Y_{F}^{m}$ is the value of the fuel mass fraction in the unburnt gas, and $Y_{F}^{e q}$ its value in the burnt gas. $Y_{F}^{m}$ is an input quantity to the combustion problem that characterizes the pre-combustion state of the reactive mixture; $Y_{F}^{e q}$ is a quantity that characterizes the post-premixed-flame state. Upstream of the deflagration front, $\tilde{c}=0$ and the mixture composition corresponds to the pure mixing solution, $Y_{F}=Y_{F}^{m}(\tilde{Z})$, with $\tilde{Z}$ the LES-filtered mixture fraction, whereas downstream of the deflagration front, $\tilde{c}=1$ and the mixture composition may be approximated by the classical Burke-Schumann equilibrium solution, $Y_{F}=Y_{F}^{e q}(\tilde{Z})$. We have: $\left(Y_{F}^{m}-Y_{F}^{e q}\right)=\tilde{Z}$ if $\tilde{Z} \leq Z_{s t}$; and $\left(Y_{F}^{m}-Y_{F}^{e q}\right)=(1-\tilde{Z}) \times Z_{s t} /\left(1-Z_{s t}\right)$, if $\tilde{Z} \geq Z_{s t}$.

The filtered-c model in Eqs. (1)-(2) has previously been implemented into FDS and tested in configurations corresponding to homogeneous, laminar or turbulent deflagrations [22]. Ref. [22] presents a detailed discussion of the grid resolution requirement of the model formulation in Eqs. (1)-(2), and of the relationship between the LES $c$-filter size $\Delta_{c}$ and the computational grid cell size $\Delta$. It can be shown that the thickness $\delta_{f}$ of the LES-filtered flame is $\delta_{f}=\Delta_{c} \sqrt{\pi / 6}\left(1+\left(16 \sqrt{6 / \pi} v_{t}\right) /\left(S c_{t} s_{L} \Delta_{c}\right)\right)^{1 / 2}$ (i.e. is of order $\left.\Delta_{c}\right)$, and the flame is correctly resolved on the computational grid for values of $\left(\Delta_{c} / \Delta\right)$ much larger than one [22]. The numerical tests performed in Ref. [22] suggest that the filtered-c model in FDS requires a filterto-grid length scale ratio equal to or greater than $4,\left(\Delta_{C} / \Delta\right) \geq 4$. 
We now turn to the description of the laminar flame speed $s_{L}$. $s_{L}$ varies with the fuel-air mass ratio (or equivalently the mixture fraction $\tilde{Z}$ ), the unburnt gas temperature $T_{u}$ and the bulk pressure $\bar{p}$. The variations of $s_{L}$ with $T_{u}$ and $\bar{p}$ are described using closure expressions proposed in Ref. [28]: $s_{L}=s_{L, \text { ref }}(\tilde{Z})\left(T_{u} / T_{u, r e f}\right)^{\gamma}\left(\bar{p} / \bar{p}_{\text {ref }}\right)^{\beta}$ where $s_{L, \text { ref }}(\tilde{Z})$ is the value of $s_{L}$ obtained at normal temperature and pressure conditions $\left(T_{u}=T_{u, r e f}=298 \mathrm{~K}\right.$ and $\left.\bar{p}=\bar{p}_{\text {ref }}=101.3 \mathrm{kPa}\right)$, and where $\gamma$ and $\beta$ are model coefficients that are functions of the local fuel-air mass ratio: $\gamma=2.18-0.8(\phi-1)$ and $\beta=-0.16+0.22(\phi-1)$ with $\phi=\left(1-Z_{s t}\right) \times \tilde{Z} / Z_{s t} /(1-\tilde{Z})$. The variations of $s_{L, \text { ref }}$ with $\tilde{Z}$ are described via an $a d-h o c$ analytical expression parametrized in terms of four input variables, called $Z_{L F L}, Z_{U F L}, Z_{s t}$ and $s_{L, s t} . Z_{L F L}$ and $Z_{U F L}$ are the values of mixture fraction $Z$ at the lower and upper flammability limits; $Z_{s t}$ and $s_{L, s t}$ are the stoichiometric values of $Z$ and $s_{L, \text { ref }}$. We use here a piecewise second-order polynomial function that vanishes at $Z_{L F L}, Z_{U F L}$, is maximum at $Z_{s t}$, and features a peak value equal to $s_{L, s t}$ [23-25].

Refs. [23-25] present a detailed discussion of the grid resolution requirement of the PPC model formulation when used in non-homogeneous configurations (i.e., configurations with variations in mixture fraction) and show that in addition to the filter-to-grid length scale ratio constraint discussed above, there is a requirement that the LES premixed flame remains thin in mixture fraction space. This requirement is quite demanding and corresponds to an important limitation of the present PPC model. To overcome this difficulty, a modified PPC formulation is proposed in Ref. [25] and is also adopted here. The modified formulation is based on a two-speed treatment $\left(s_{L}^{*}, s_{L}^{* *}\right)$ where $s_{L}^{*}$ is used in Eq. (1) and $s_{L}^{* *}$ in Eq. (2), and where $s_{L}^{*}=s_{L}^{* *}=s_{L}$ within the flammable regions, while $s_{L}^{* *}<s_{L}<s_{L}^{*}$ near the edges (i.e., at locations

where $\tilde{Z}$ is close to $Z_{L F L}$ or $Z_{U F L}$ ). This scheme has proven successful at eliminating spurious premixed burning near the flammability limits and at providing a clean description of the burn out phase after premixed burning is completed (see Ref. [25] for additional details).

\section{Diffusion Flame Modeling}

We adopt in the present study the combustion modeling framework proposed in FDS Version 5 [21]. This framework is limited to a description of non-premixed burning and corresponds to a one-step or two-step global combustion model, with or without flame extinction due to air vitiation. We limit our discussion herein to the one-step version of the combustion model. We start from the global combustion equation:

$$
\mathrm{C}_{n} \mathrm{H}_{m}+v_{\mathrm{O}_{2}} \mathrm{O}_{2} \rightarrow\left(n-v_{\mathrm{CO}}-v_{\text {soot }}\right) \mathrm{CO}_{2}+\frac{m}{2} \mathrm{H}_{2} \mathrm{O}+v_{\mathrm{CO}} \mathrm{CO}+v_{\text {soot }} \mathrm{C} \quad(\mathrm{R} 1)
$$

where $v_{\mathrm{O}_{2}}=\left(n+(m / 4)-\left(v_{\mathrm{CO}} / 2\right)-v_{\text {soot }}\right)$ and where combustion products include $\mathrm{CO}_{2}, \mathrm{H}_{2} \mathrm{O}$, and also $C O$ and soot (treated as pure carbon). The stoichiometric coefficients in reaction $(R 1)$ are simply obtained from the fuel chemical composition and user-specified carbon monoxide and soot yields.

In FDS, the mixture composition is described using two reactive scalars, called $Z_{1}$ and $Z_{2}$. These scalars are based on the following decomposition of carbon mass:

$$
Z=\underbrace{Y_{C_{n} H_{m}}}_{Z_{1}}+\underbrace{\left(\frac{W_{C_{n} H_{m}}}{n W_{C O_{2}}}\right) Y_{C O_{2}}+\left(\frac{W_{C_{n} H_{m}}}{n W_{C O}}\right) Y_{C O}+\left(\frac{W_{C_{n} H_{m}}}{n W_{\text {soot }}}\right) Y_{\text {soot }}}_{Z_{2}}
$$

where $Z_{1}$ represents the carbon mass fraction contained in the fuel, and $Z_{2}$ the carbon mass fraction contained in $\mathrm{CO}_{2}, \mathrm{CO}$ and soot, and where $Y_{k}$ and $W_{k}$ are the mass fraction and molecular weight of species $k$. It can be shown that the entire mixture composition can be reconstructed from the knowledge of $Z_{1}$ and $Z_{2}$ via state relationships. 
The corresponding governing equations are:

$$
\left.\begin{array}{l}
\frac{\partial}{\partial t}\left(\bar{\rho} \tilde{Z}_{1}\right)+\frac{\partial}{\partial x_{i}}\left(\bar{\rho} \tilde{u}_{i} \tilde{Z}_{1}\right)=\frac{\partial}{\partial x_{i}}\left(\bar{\rho} \frac{v_{t}}{S c_{t}} \frac{\partial \tilde{Z}_{1}}{\partial x_{i}}\right)-\overline{\dot{\omega}_{R 1}^{\prime \prime \prime}} \\
\frac{\partial}{\partial t}\left(\bar{\rho} \tilde{Z}_{2}\right)+\frac{\partial}{\partial x_{i}}\left(\bar{\rho} \tilde{u}_{i} \tilde{Z}_{2}\right)=\frac{\partial}{\partial x_{i}}\left(\bar{\rho} \frac{v_{t}}{S c_{t}} \frac{\partial \tilde{Z}_{2}}{\partial x_{i}}\right)+\overline{\dot{\omega}_{R 1}^{\prime \prime \prime}}
\end{array}\right\}
$$

where $\overline{\dot{\omega}_{R 1}^{\prime \prime \prime}}$ is the mass reaction rate of the global combustion reaction (R1). In FDS, combustion is treated using a closure expression known as the Eddy Dissipation Concept model [29], $\overline{\dot{\omega}_{R 1}^{\prime \prime \prime}}=\overline{\dot{\omega}_{d}^{\prime \prime \prime}}$; we write:

$\overline{\dot{\omega}_{d}^{\prime \prime \prime}}=[1-F E F] \times \bar{\rho} \times \frac{\min \left(\tilde{Y}_{F} ; \tilde{Y}_{O_{2}} / r_{s}\right)}{\tau}$

where $r_{s}$ is the stoichiometric oxygen-to-fuel mass ratio, $\tau$ a characteristic combustion time scale, and where FEF is a flame extinction factor that takes values 0 or 1 , and is determined according to a diffusion flame extinction model. Consistent with the classical idea that in the absence of flame extinction, chemistry is fast, the time scale $\tau$ is simply set equal to the computational time step, $\tau=\Delta t$. The flame extinction model describes the effects of air vitiation (i.e. air mixed with recirculating combustion products; see Refs. [21] and [30] for additional details): FEF $=0$ at flame locations that are well-ventilated, whereas FEF =1 at locations that are supplied with super-critical levels of vitiated air.

\section{Coupling Interface}

We now turn to a description of the coupling interface between the premixed and non-premixed flame models discussed above. The interface formulation provides a generalized expression for the global combustion reaction $\overline{\dot{\omega}_{R 1}^{\prime \prime \prime}}$. This generalized expression is based on an identification of the locally dominant combustion mode (premixed versus non-premixed) using the concept of a LES-resolved flame index FI [18-19]. Following Ref. [18], we define the flame index as:

$F I=\frac{1}{2}\left(\frac{\nabla \tilde{Y}_{F} \cdot \nabla \tilde{Y}_{O_{2}}}{\left|\nabla \tilde{Y}_{F}\right| \times\left|\nabla \tilde{Y}_{O_{2}}\right|}+1\right)$

where $\tilde{Y}_{F}$ and $\tilde{Y}_{O_{2}}$ are the grid-resolved fuel and oxygen mass fractions (determined as functions of $\tilde{Z}_{1}$ and $\tilde{Z}_{2}$ via state relationships). Note that this expression differs slightly from that in Ref. [18]: the FIexpression in Ref. [18] includes a subgrid-scale contribution; this contribution is neglected in Eq. (7).

As seen in Eq. (7), $F I$ is a non-dimensional field quantity that varies between 0 and 1: inert mixing between cross-diffusing fuel and oxygen corresponds to $F I=0$; a diffusion flame configuration in which fuel and oxygen penetrate the diffusive/reactive layer from opposite directions also corresponds to $F I=0$; in contrast, a premixed flame configuration corresponds to $F I=1$. In regions where the fuel or oxygen mass is homogeneously distributed (i.e. in regions where $\nabla \tilde{Y}_{F}=0$ or $\nabla \tilde{Y}_{O_{2}}=0$ ), FI is set to 0 .

We adopt in the following the PPC closure model of Ref. [18] and describe $\overline{\dot{\omega}_{R 1}^{\prime \prime \prime}}$ as a weighted average between the premixed and non-premixed contributions, using FI as a weight coefficient:

$\overline{\dot{\omega}_{R 1}^{\prime \prime \prime}}=F I \times \overline{\dot{\omega}_{p}^{\prime \prime \prime}}+(1-F I) \times f_{i g n} \times \overline{\dot{\omega}_{d}^{\prime \prime \prime}}$ 
where $f_{\text {ign }}$ is an $a d$ hoc ignition factor. $f_{\text {ign }}$ is introduced in Eq. (8) so that the diffusion flame model remains inactive wherever inert mixing is taking place $\left(f_{\text {ign }}=0\right.$ when $\left.\tilde{c}=0\right)$, and is only activated as a postpremixed-flame event ( $f_{\text {ign }}=1$ when $\tilde{c}=1$ ). We use the expression: $f_{\text {ign }}=0.5+0.5 \tanh ((\tilde{c}-0.6) / 0.05)$.

Enhanced by the coupling scheme in Eqs. (7)-(8), Eqs. (1)-(6) correspond to a combustion model with a partially-premixed combustion capability. This model has been implemented in an in-house version of FDS (Version 5). We focus in the remainder of the paper on a series of simulations aimed at evaluating the overall performance of the PPC/FDS model against previously obtained experimental data. The test configuration corresponds to explosive combustion in a confined environment and features significant bulk pressure variations. Since much of the evaluation of the PPC/FDS model performance will rely on comparisons between measured and simulated pressure time histories, we first address in the next Section issues associated with the bulk pressure algorithm of FDS. We then proceed in the Section that follows to a discussion of the validation study.

\section{Bulk Pressure Modeling}

We start from the bulk pressure equation [21]:

$$
\frac{d \bar{p}}{d t}=\frac{-\oiint_{C S} \tilde{u}_{j} n_{j} d S+\iiint_{C V} \frac{\gamma-1}{\bar{p}}\left(\frac{\partial}{\partial x_{i}}\left(\bar{\rho} \frac{v_{t}}{S c_{t}} c_{p} \frac{\partial \tilde{T}}{\partial x_{i}}\right)-\frac{\partial \overline{\dot{q}_{R, j}^{\prime \prime}}}{\partial x_{i}}+\overline{\dot{q}_{c}^{\prime \prime \prime}}\right) d V}{\iiint_{C V} \frac{d V}{\bar{p}}}
$$

where $c_{p}$ is the specific heat (at constant pressure), $\gamma$ the ratio of specific heats, $\tilde{T}$ the temperature, $\overline{\dot{q}_{R, j}^{\prime \prime}}$ the $x_{j}$-component of the radiation heat flux vector (in units of $\mathrm{W} / \mathrm{m}^{2}$ ), and $\overline{\dot{q}_{c}^{\prime \prime \prime}}$ the combustion heat release rate $\left(\mathrm{W} / \mathrm{m}^{3}\right), \overline{\dot{q}_{c}^{\prime \prime \prime}}=\overline{\dot{\omega}_{R 1}^{\prime \prime \prime}} \times \Delta H_{F}$ with $\Delta H_{F}$ the heat of combustion (per unit mass of fuel). The integral terms in Eq. (9) are calculated as volume integrals over the arbitrary control volume $C V$, or surface integrals over its control surface $C S$ ( $n_{j}$ is the $x_{j}$-component of the unit vector normal to $C S$ and pointing outward). In the following, $C V$ denotes the explosion chamber. The first term in the numerator of the RHS of Eq. (9) represents the effects of convective transport across vents: an inflow of mass $\left(\tilde{u}_{j} n_{j}<0\right)$ tends to increase the compartment pressure, whereas an outflow $\left(\tilde{u}_{j} n_{j}>0\right)$ decreases it. The second term represents the effects of convective/radiative heat transfer across CS (predominantly wall heat losses) as well as those of combustion: heat losses tend to decrease the pressure whereas heat release tends to increase it.

Eq. (9) is an ordinary differential equation that can in principle be used as a closure model for $\bar{p}$. It turns out, however, that this equation is numerically stiff and requires special care for numerical integration (see for instance past studies of the equation for pressure conducted in the context of zone modeling [31-33]). This point is overlooked in FDS, where the same explicit predictor-corrector time integration scheme is used for the flow/fire variables and for $\bar{p}$ [21]. In the absence of a proper treatment, the integration of Eq. (9) can lead to the development of numerical instabilities and to a computational crash.

This analysis was confirmed in a series of FDS tests that also revealed that numerical problems are limited to configurations with vents and do not occur in sealed compartments $\left(\oiint_{C S} \tilde{u}_{j} n_{j} d S=0\right)$. This is an interesting result since in the presence of vents, the bulk pressure equation may be bypassed entirely by adopting a classical zone modeling strategy. For instance, assuming steady state and using a Bernoulli expression for the outflow velocities, $\tilde{u}_{j}=\left(2 \Delta \bar{p}_{e q} / \bar{\rho}\right)^{1 / 2}$ wherever $\tilde{u}_{j} n_{j}>0$, with $\Delta \bar{p}_{e q}=\left(\bar{p}-\bar{p}_{\infty}\right)$ the compartment over-pressure and $\bar{p}_{\infty}$ the external atmospheric pressure, one obtains: 


$$
\Delta \bar{p}_{e q} \approx \frac{-\oiint_{C S, \tilde{u}_{j} n_{j}<0} \tilde{u}_{j} n_{j} d S+\iiint_{C V} \frac{\gamma-1}{\bar{p}}\left(\frac{\partial}{\partial x_{i}}\left(\bar{\rho} \frac{v_{t}}{S c_{t}} c_{p} \frac{\partial \tilde{T}}{\partial x_{i}}\right)-\frac{\partial \overline{\dot{q}_{R, j}^{\prime \prime}}}{\partial x_{i}}+\overline{\dot{q}_{c}^{\prime \prime \prime}}\right) d V}{\oiint_{C S, \tilde{u}_{j} n_{j}>0}\left(\frac{2}{\bar{\rho}}\right)^{1 / 2} d S}
$$

where the surface integrals over the vent openings of $C S$ are conditioned on inflow or outflow state.

The pressure algorithm in FDS has been modified according to the observations above. We assume a scenario in which the fire compartment is initially sealed, and the pressure $\bar{p}$ rises, until a vent bursts open at time $t=t_{\mathrm{O}}$ because a critical value of $\bar{p}$ has been reached. The following scheme is proposed: (1) for $0 \leq$ $t \leq t_{\mathrm{O}}$ (sealed configuration), calculate $\bar{p}(t)$ from Eq. (9) and store the value $\bar{p}\left(t=t_{O}\right)$; (2) for $t_{\mathrm{O}}<t$ (vented configuration), use Eq. (10) and write:

$$
\left(\bar{p}-\bar{p}_{\infty}\right)=\left(\bar{p}\left(t=t_{O}\right)-\bar{p}_{\infty}\right) \times \exp \left(-\left(t-t_{O}\right) / \tau\right)+\Delta \bar{p}_{e q} \times\left(1-\exp \left(-\left(t-t_{O}\right) / \tau\right)\right)
$$

where $\tau$ is a relaxation time scale assumed to be fast ( $\tau$ is set to a value that is a few times larger than the computational time step). Eq. (11) allows for a smooth transition from the ordinary differential equation model in Eq. (9) to the quasi-steady state expression in Eq. (10).

Note that while the modifications proposed in Eqs. (9)-(11) provide a valuable solution to the problem of calculating the bulk pressure in FDS, other problems remain and have yet to be resolved. For instance, the velocity field in FDS remains insensitive to the over-pressure $\left(\bar{p}-\bar{p}_{\infty}\right)$ and preliminary attempts to modify the velocity boundary conditions at open flow boundaries (in order to fully couple the velocity algorithm to that of $\bar{p}$ ) have proven unsuccessful. Because of this unresolved problem, the outflow velocities at vent openings in an over-pressurized compartment are likely to be significantly underestimated.

\section{NUMERICAL SIMULATIONS OF EXPLOSIVE COMBUSTION}

Our in-house version of FDS Version 5, enhanced by both a partially-premixed combustion model and a modified bulk pressure algorithm, is now evaluated via detailed comparisons with an experimental database previously developed by FM Global Research. The configuration corresponds to controlled ignition followed by explosive combustion in an enclosure filled with vertically-stratified mixtures of propane in air, both with and without venting, and with and without obstacles [34-36]. This database was originally developed for analysis of explosion hazards associated with flammable liquid spills or releases of heavy flammable vapors in enclosures.

\section{Configuration}

The FM Global explosion chamber is a rectangular-shaped $63.7 \mathrm{~m}^{3}$ enclosure with a $4.57 \times 4.57 \mathrm{~m}^{2}(15 \times$ $\left.15 \mathrm{ft}^{2}\right)$ square base and a $3.05 \mathrm{~m}(10 \mathrm{ft})$ height. The walls of the enclosure are made of $38 \mathrm{~mm}(1.5 \mathrm{in})$ plywood panels that are steel-faced $(0.41 \mathrm{~mm}$, or $0.016 \mathrm{in})$, while the floor is made of concrete material. The enclosure is made as tight as possible by covering all the joints with a bead of silicone sealant. Rectangular openings $\left(0.51 \times 1.12 \mathrm{~m}^{2}\right.$ or $\left.20 \times 44 \mathrm{in}^{2}\right)$ are available on the roof of the chamber for explosion venting. A few of the available roof vents are used during some tests: the vents are then covered with a sheet of polyethylene that bursts open at known over-pressure levels. In addition, a significant number of tests are conducted with obstacles that are introduced to study the effect of blockages; the obstacle array corresponds to $0.76 \times 0.76 \mathrm{~m}^{2}\left(2.5 \times 2.5 \mathrm{ft}^{2}\right)$ steel plates installed horizontally in a checkered pattern $0.46 \mathrm{~m}$ $(1.5 \mathrm{ft})$ above the floor; the array provides a $50 \%$ blockage to vertical flow/flame expansion (but less resistance to horizontal motions). 


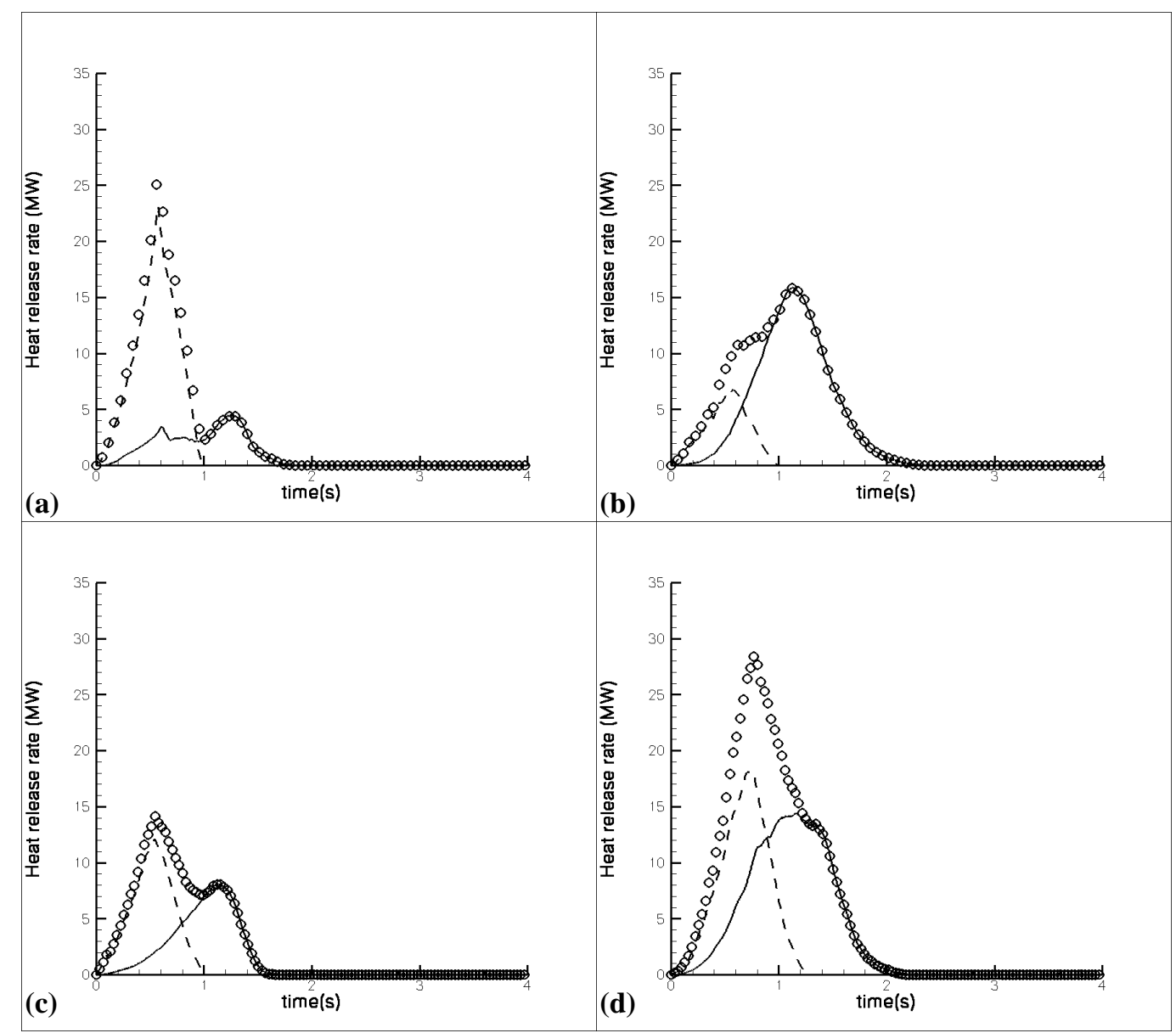

Fig. 3. Time variations of the simulated heat release rate. The plots show the total heat release rate (circles) as well as its premixed (dashed line) and diffusion flame (solid line) components. (a) case 6; (b) case 26;

(c) case 7; (d) case 14.

We focus in the present study on 4 experimental cases: case 6 that is unvented and without obstacle; case 26 that is unvented and with obstacles; case 7 that is vented ( 1 roof vent) and without obstacle; case 14 that is vented ( 1 roof vent) and with obstacles. In all cases, a quasi-one-dimensional, vertically-stratified layer of flammable gas is produced prior to ignition by controlled, floor-level, low-velocity, injection of propane. The mixture composition is monitored in time by a gas analysis system. Ignition is triggered in the center of the chamber using an arrangement known as a "Jacob’s ladder".

The list of experimental diagnostics include video observations of the flames and measurements of the time history of the chamber pressure. Because of the presence of uncontrolled leaks, the pressure measurements are corrected to provide an estimate of the pressure that would have been obtained in the absence of leaks and wall heat losses [34-36]. This corrected pressure will be the main diagnostic used for comparisons with FDS results.

The FDS computational domain corresponds to the explosion chamber. The simulations start at ignition time and use the (case-dependent) measured distribution of propane in air for initial conditions. The computational grid corresponds to a uniform rectangular mesh; the size of the mesh is adjusted to adequately resolve the floor-level flammable portion of the propane-air layer; the mesh corresponds to cubic grid cells with a $\Delta$ spacing: $\Delta=2.5 \mathrm{~cm}$ in cases 6 and $14, \Delta=1.25 \mathrm{~cm}$ in case 7 and $\Delta=0.8 \mathrm{~cm}$ in case 26. The flame speed model parameters are: $Z_{L F L}=0.032, Z_{U F L}=0.153, Z_{s t}=0.06$ and $s_{L, s t}=0.44 \mathrm{~m} / \mathrm{s}$. The filter-to-grid length scale ratio is equal to $5,\left(\Delta_{C} / \Delta\right)=5$. Based on trial and error, the flame wrinkling factor is fixed at a relatively high values, $\Xi=4$. Simulations are performed on a multi-processor Linux cluster available at the University of Maryland, using the parallel MPI-based version of FDS. 


\section{Results}

The simulations provide valuable insights into the transient combustion dynamics that follow ignition. Fig. 3 presents the time variations of the simulated spatially-averaged heat release rate as well as those of its premixed and diffusion flame components, as obtained using the PPC formulation (Eq. (8)). The heat release rate is maximum shortly after ignition (at $t=0.5 \mathrm{~s}$ in case 6 , at $t=1.2 \mathrm{~s}$ in case 26) and reaches a peak value that ranges from 15 MW (cases 7 and 26) to more than 25 MW (cases 6 and 14). In all cases, the combustion phase is short and lasts between 1.5 and $2 \mathrm{~s}$; combustion ceases because of fuel depletion. An analysis of the different simulations reveals that the flame expands from the centrally-located ignition point in both horizontal and (upward) vertical directions. The horizontal spread is associated with the premixed flame (the flash fire), whereas the vertical spread is associated with a buoyancy-driven diffusion flame (a fireball). The intensity of both flames depends strongly on the state of the propane-air mixing field found at ignition time [34-36]. For instance, in case 6, the bulk of the propane cloud is flammable fuel-lean and combustion is predominantly premixed (Fig. 3(a)). In contrast, in case 26, the propane cloud features a large ultra-rich layer and combustion is in that case predominantly non-premixed (Fig. 3(b)). Finally, in cases 7 and 14, the bulk of the propane cloud is flammable fuel-rich/fuel-lean and combustion is partiallypremixed (Figs. 3(c)-(d)). In all cases, premixed burning peaks when the deflagration impinges on the vertical side walls of the chamber, while diffusion burning peaks when fuel depletion effects become dominant.

Fig. 4 compares the experimental and simulated time histories of bulk pressure. As mentioned earlier, the experimental data are corrected for the presence of leaks and wall heat losses. In cases 6 and 26 (unvented), the pressure increases to more than $60 \mathrm{kPa}$ and reaches a plateau once the combustion is completed (Figs. 4(a)-(b)). The good agreement between experimental data and numerical results when comparing the timing of the pressure increase suggests that the rate of combustion is reasonably well predicted (in Refs. [34-36], the turbulent flame speed that characterizes the burning intensity of the deflagration wave is estimated to be $1.75 \pm 0.25 \mathrm{~m} / \mathrm{s}$ ). The fair agreement when comparing the post-combustion pressure levels suggests that the total amount of propane mass consumed is predicted less accurately (within 20-30\%). In cases 7 and 14 (vented), the pressure variations feature two peaks (Figs. 4(c)-(d)): the first peak is associated with the sudden opening of the roof vent (at $\bar{p} \approx 3 \mathrm{kPa}$ ); the second peak corresponds to the timing of maximum heat release rate (Fig. 3(c)-(d)). The first pressure peak is well predicted in case 14, but predicted with some delay in case 7; the magnitude of the second peak is under-predicted in both cases, which suggests that the peak intensity of the heat release rate might also be under-predicted. As pointed out in the Bulk Pressure Modeling Section, the vent outflow velocities are not correctly described in FDS, which will lead to incorrect flow/flame predictions in the post-vent-opening phase in vented explosion scenarios.

\section{CONCLUSION}

The present study is aimed at adapting current large eddy simulation capabilities to a description of lowpressure explosions in fuel vapor clouds, with an emphasis on scenarios featuring delayed ignition followed by coupled deflagration and diffusion burning. The proposed model formulation is based on a filtered reaction progress variable approach to treat premixed combustion, the Eddy Dissipation Concept for nonpremixed combustion, and the flame index concept to provide a coupling interface. The partially-premixed combustion (PPC) model is implemented in the Fire Dynamics Simulator (Version 5) developed by the National Institute of Standards and Technology. Its performance is evaluated in a validation study using an experimental database previously developed by FM Global Research; the database corresponds to explosive combustion tests in an enclosure filled with vertically-stratified mixtures of propane in air, both with and without venting.

The experimental database is well-suited to testing the PPC model since it includes some cases in which combustion is predominantly premixed and other cases in which it is essentially non-premixed. The unvented compartment cases develop bulk over-pressures up to approximately $60 \mathrm{kPa}(9 \mathrm{psi})$; the vented cases develop over-pressures up to $3 \mathrm{kPa}(0.4 \mathrm{psi})$. These pressurized combustion scenarios present a particular challenge to the bulk pressure algorithm in FDS which has robustness and accuracy issues, in 


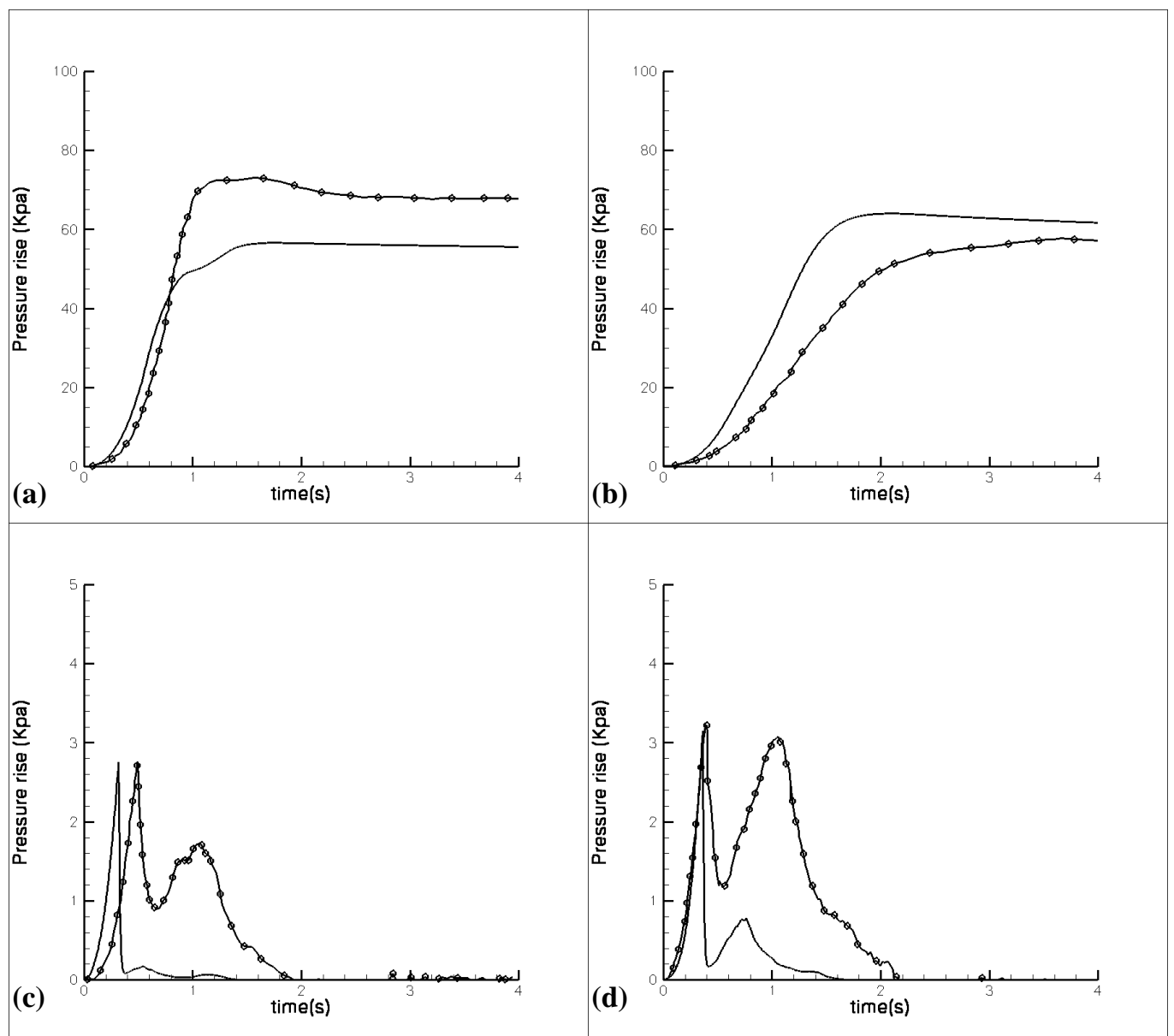

Fig. 4. Time variations of the bulk compartment pressure. Comparisons between experimental data (circles) and numerical results (solid line). (a) case 6; (b) case 26; (c) case 7; (d) case 14.

particular in vented configurations. The FDS bulk pressure algorithm has been modified in the present study in order to allow detailed comparisons between measured and simulated pressure time histories. Overall, the comparison between numerical results and experimental data ranges from fair to good and confirms the feasibility of a numerical treatment of explosive combustion. Future work will focus on some unresolved problems in FDS for applications to explosion scenarios, and in particular the problem of coupling the open-flow velocity boundary conditions to the bulk pressure algorithm.

\section{ACKNOWLEDGMENTS}

This work was supported in part by the U.S. National Institute of Standards and Technology, Building and Fire Research Laboratory. This work also benefited from fruitful interactions and sharing of data with Dr. F. Tamanini from FM Global Research. The support of Dr. Robert Bill from FM Global Research is also gratefully acknowledged.

\section{REFERENCES}

[1] Strehlow, R.A., (1973) Unconfined Vapor Cloud Explosions - an Overview, Proceedings of the Combustion Institute 14:1189-1200.

[2] Baker, W.E., and Tang, M.J., Gas, Dust and Hybrid Explosions, Elsevier, 1991.

[3] Bradley, D., Cresswell, T.M., and Puttock, J.S., (2001) Flame Acceleration due to Flame-Induced Instabilities in Large-Scale Explosions, Combustion and Flame 124:551-559. http://dx.doi.org/10.1016/S0010-2180(00)00208-X 
[4] Zalosh, R., "Explosion Protection,” The SFPE Handbook of Fire Protection Engineering (3 ${ }^{\text {rd }}$ ed), DiNenno P.J. (ed.), National Fire Protection Association, Quincy, MA 02269, 2002, p. 3/402.

[5] Guidelines for Evaluating the Characteristics of Vapor Cloud Explosions, Flash Fires, and BLEVEs, Center for Chemical Process Safety, AIChE, 1998.

[6] Molkov, V., Makarov, D., and Grigorash, A., (2004) Cellular Structure of Explosion Flames: Modeling and Large Eddy Simulation, Combustion Science and Technology 176:851-865. http://dx.doi.org/10.1080/00102200490428495

[7] Makarov, D.V., and Molkov, V.V., (2004) Modeling and Large Eddy Simulation of Deflagration Dynamics in a Closed Vessel, Combustion, Explosion and Shock Waves 40:136-144. http://dx.doi.org/10.1023/B:CESW.0000020133.28696.fb

[8] Molkov, V., Makarov, D., and Puttock, J., (2006) The Nature and Large Eddy Simulation of Coherent Deflagrations in a Vented Enclosure-Atmosphere System, Journal of Loss Prevention in the Process Industries 19:121-129. http://dx.doi.org/10.1016/j.jlp.2005.05.006

[9] Makarov, D., Verbecke, F. and Molkov, V., (2007) Numerical Analysis of Hydrogen Deflagration Mitigation by Venting through a Duct, Journal of Loss Prevention in the Process Industries 20:433-438. http://dx.doi.org/10.1016/j.jlp.2007.04.022

[10] Makarov, D., Molkov, V., and Gostintsev, Yu, (2007) Comparison between RNG and Fractal Combustion Models for LES of Unconfined Explosions, Combustion Science and Technology 179:401-416. http://dx.doi.org/10.1080/00102200600835626

[11] Cant, R.S., Dawes, W.N., and Savill, A.M., (2004) Advanced CFD and Modeling of Accidental Explosions, Annual Review Fluid Mechanics 36:97-119. http://dx.doi.org/10.1146/annurev.fluid.36.050802.121948

[12] Makhviladze, G.M., Roberts, J.P., and Yakush, S.E., "Modelling the Fireballs from Methane Releases," Fire Safety Science -- Proceedings of the Fifth International Symposium, International Association for Fire Safety Science, 1997, pp. 213-224.

[13] Makhviladze, G.M., Roberts, J.P., and Yakush, S.E., (1998) Numerical Modelling of Fireballs from Vertical Releases of Fuel Gases, Combustion Science and Technology 132:199-223. http://dx.doi.org/10.1080/00102209808952015

[14] Makhviladze, G.M., Roberts, J.P., and Yakush, S.E., (1999) Combustion of Two-Phase Hydrocarbon Fuel Clouds Released into the Atmosphere, Combustion and Flame 118:583-605. http://dx.doi.org/10.1016/S0010-2180(99)00026-7

[15] Makhviladze, G.M., Roberts, J.P., and Yakush, S.E., "Modelling and Scaling of Fireballs from Single -and Two-Phase Hydrocarbon Releases," Fire Safety Science -- Proceedings of the Sixth International Symposium, International Association for Fire Safety Science, 2000, pp. 1125-1136.

[16] Makhviladze, G.M., and Yakush, S.E., "Modelling of Fires Following Bursts of Pressurized Fuel Tanks," Fire Safety Science -- Proceedings of the Seventh International Symposium, International Association for Fire Safety Science, 2003, pp. 643-654.

[17] Müller, C.M., Breitbach, H., and Peters, N., (1994) Partially Premixed Turbulent Flame Propagation in Jet Flames, Proceedings of the Combustion Institute 25:1099-1106.

[18] Domingo, P., Vervisch, L., and Bray, K., (2002) Partially Premixed Flamelets in LES of Nonpremixed Turbulent Combustion, Combustion Theory and Modelling 6:529-551. http://dx.doi.org/10.1088/1364-7830/6/4/301

[19] Vervisch, L., Hauguel, R., Domingo, P., and Rullaud, M., (2004) Three Facets of Turbulent Combustion Modelling: DNS of Premixed V-Flame, LES of Lifted Nonpremixed Flame and RANS of Jet-Flame, Journal of Turbulence 5:004.

[20] McGrattan, K.B., Floyd, J.E., Forney, G.P., Baum, H.R., and Hostikka, S., "Improved Radiation and Combustion Routines for a Large Eddy Simulation Fire Model," Fire Safety Science -- 
Proceedings of the Seventh International Symposium, International Association for Fire Safety Science, 2003, pp. 827-838.

[21] McGrattan, K.B., Hostikka, S., Floyd, J.E., Baum, H.R., and Rehm, R.G., "Fire Dynamics Simulator (Version 5) - Technical Reference Guide,” National Institute of Standards and Technology Report NIST Special Publication 1018-5, Gaithersburg, MD, USA, 2007.

[22] Williamson, J., McGill, J. and Trouvé, A., "Large Eddy Simulation Modeling of Turbulent Deflagrations," Fire Safety Science -- Proceedings of the Eigth International Symposium, International Association for Fire Safety Science, 2005, pp. 1375-1386.

[23] Wiley, J., and Trouvé, A., Large Eddy Simulation of Flash Fires Following Ignition of a Fuel Vapor Cloud, Proceedings of 5th U.S. Combustion Meeting, San Diego, CA, 2007.

[24] Wiley, J., and Trouvé, A., Large Eddy Simulation of Ignition and Transient Combustion in Fuel Vapor Clouds, Proceedings of $5^{\text {th }}$ Intl. Seminar on Fire and Explosion Hazards, Edinburgh, U.K., 2007.

[25] Wiley, J., "Numerical Simulation of Ignition and Transient Combustion in Fuel Vapor Clouds,” MS Thesis, University of Maryland, 2007.

[26] Boger, M., and Veynante, D., "Large Eddy Simulation of a Turbulent Premixed V-Shaped Flame," Advances in Turbulence, Dopazo C. (ed.), Cimne, Barcelona, 2000, p. 449.

[27] Boger, M., "Modélisation de Sous-Maille pour la Simulation aux Grandes Echelles de la Combustion Turbulente Prémélangée,” PhD Thesis, Ecole Centrale Paris, France, 2000.

[28] Turns, S.R., An Introduction to Combustion - Concepts and Applications (2 ${ }^{\text {nd }}$ ed.), McGraw-Hill, 2000.

[29] Magnussen, B.F, and Hjertager, B.H., (1976) On Mathematical Modeling of Turbulent Combustion with Special Emphasis on Soot Formation and Combustion, Proceedings of the Combustion Institute 16:719-729.

[30] Hu, Z., Utiskul, Y., Quintiere, J.G., and Trouvé A., (2007) Towards Large Eddy Simulations of Flame Extinction and Carbon Monoxide Emission in Compartment Fires, Proceedings of the Combustion Institute 31:2537-2545. http://dx.doi.org/10.1016/j.proci.2006.08.053.

[31] Quintiere, J.G., (1984) Fundamentals of Enclosure Fire Zone Models, Journal of Fire Protection Engineering 1:99-119. http://dx.doi.org/10.1177/104239158900100302

[32] Rehm, R.G., and Forney, G.P., (1994) The Pressure Equations in Zone-Fire Modeling, Fire Science and Technology 14:61-73.

[33] Forney, G.P., and Moss, W.F., (1994) Analyzing and Exploiting Numerical Characteristics of Zone Fire Models, Fire Science and Technology 14:49-60.

[34] Tamanini, F., and Chaffee, J.L., (2000) Mixture Reactivity in Explosions of Stratified Fuel/Air Layers, Process Safety Progress 19:219-227. http://dx.doi.org/10.1002/prs.680190407

[35] Tamanini, F., Partial-Volume Deflagrations - Characteristics of Explosions in Layered Fuel/Air Mixtures, Proceedings of $3^{\text {rd }}$ Intl. Seminar on Fire and Explosion Hazards, Edinburgh, U.K., 2001, p. 103.

[36] Tamanini, F., private communication. 